\title{
Bicontinuous Nanotubular Graphene-Polypyrrole Hybrid for High Performance Flexible Supercapacitors
}

\section{Hamzeh Kashani}

WPI Advanced Institute for Materials Research, Tohoku University, Sendai, 980-8577, Japan.

Email: hkashani3073@yahoo.com

\section{Luyang Chen}

WPI Advanced Institute for Materials Research, Tohoku University, Sendai, 980-8577, Japan. Email: chenly@wpi-aimr.tohoku.ac.jp

\section{Yoshikazu Ito}

WPI Advanced Institute for Materials Research, Tohoku University, Sendai, 980-8577, Japan.

Email: ito@wpi-aimr.tohoku.ac.jp

\section{Jiuhui Han}

WPI Advanced Institute for Materials Research, Tohoku University, Sendai, 980-8577, Japan.

Email: hanjh08@gmail.com

\section{Akihiko Hirata}

WPI Advanced Institute for Materials Research, Tohoku University, Sendai, 980-8577, Japan.

Email: hirata@wpi-aimr.tohoku.ac.jp

\section{Mingwei Chen}

WPI Advanced Institute for Materials Research, Tohoku University, Sendai, 980-8577, Japan.

CREST, Japan Science and Technology Agency, Saitama, 332-0012, Japan.

Corresponding author: Mingwei Chen, Email: mwchen@wpi-aimr.tohoku.ac.jp 


\title{
Bicontinuous Nanotubular Graphene-Polypyrrole Hybrid for High Performance Flexible Supercapacitors
}

\author{
Hamzeh Kashani $^{\mathrm{a}}$, Luyang Chen ${ }^{\mathrm{a}}$, Yoshikazu Ito ${ }^{\mathrm{a}}$, Jiuhui Han ${ }^{\mathrm{a}}$, Akihiko Hirata ${ }^{\mathrm{a}}$, Mingwei \\ Chen $^{\mathrm{a}, \mathrm{b}, *}$ \\ a WPI Advanced Institute for Materials Research, Tohoku University, Sendai 980-8577, \\ Japan. \\ b CREST, Japan Science and Technology Agency, Saitama 332-0012, Japan \\ *Corresponding author: Mingwei Chen, E-mail: mwchen@wpi-aimr.tohoku.ac.jp
}

\begin{abstract}
Conductive polymers, particularly polypyrrole (PPy), are emerging as promising electrode materials for flexible supercapacitors because of their high pseudocapacitance, low density, high mechanical flexibility and low material costs. However, the practical implementations of PPy based supercapacitors have been prevented by the poor charge/discharge rate capability and low cycle stability. In this study we report a novel three dimensional interconnected nanotubular graphene-PPy (nt-GPPy) hybrid by incorporating PPy into highly conductive and stable nanoporous graphene. The bicontinuous nanotubular hybrid material with a large specific surface area and high conductivity demonstrates significant enhancement in supercapacitance performance of PPy in terms of high specific capacitance, excellent cycling stability and high rate capability.
\end{abstract}

Keywords

Nanoporous graphene; Polypyrrole; Supercapacitor; Nanoporous metals 


\section{Introduction}

Among electrochemical energy storage devices, electrochemical supercapacitors offer several merits including high energy density, high power density and long cycle stability, which are expected to bridge a gap between secondary batteries with a low power density and conventional capacitor with a low energy density [1-5]. Based on the materials of the electrodes, the mechanisms of energy storage in electrochemical supercapactitors predominately include two ways: the electric double-layer capacitance (EDLC) and pseudocapacitance. Commonly, pseudocapacitance by a surface faradic process can be realized by fast and reversible redox reactions of transition metal oxides, such as $\mathrm{Ni}(\mathrm{OH})_{2}, \mathrm{Co}_{3} \mathrm{O}_{4}, \mathrm{RuO}_{2}, \mathrm{MnO}_{2}$ and $\mathrm{NiO}$, and

conductive polymers, including polypyrrole (PPy), polyaniline and poly(3,4ethylenedioxythiophene), while porous carbon and its derivative materials with a large specific surface area are mainly used as the electrodes of EDLC supercapacitors [5-16]. The demands of light weight and flexible energy storage devices for portable electronics have stimulated tremendous interests to develop new electrode materials. Conductive polymers, particularly PPy, are emerging as promising candidates because of the low mass density, mechanical flexibility, high pseudocapacitance and low material costs $[15,16]$. However, the practical implementations of PPy based supercapacitors have been prevented by the poor charge/discharge rate capability and low cycle stability [17-21]. To overcome these challenges, many PPy based composites have recently been developed by using macro/nanoporous carbon materials and porous metals as the reinforcements, aiming at the improvement in cycle stability and rate capability [21-26]. In those composites, porous supports offer a three-dimensional conductive pathway, facilitating charge transport effectively, and micro- and nano-size pore channels serve as reservoirs of electrolytes for short ion diffusion distance and low resistance. Additionally, nanoporous architectures supply a large effective surface area, benefiting high specific capacitance. Although high rate capability 
and stability of the nanoporous PPy composites have been the recent topic of intense studies, the overall specific or volumetric capacitances based on the total mass or volume of the composite electrodes are usually very low because of the large volumetric and/or mass fraction of the porous reinforcements and inefficient utilization of the active material limited by low effective specific surface area, which produce insuperable problems for practical applications.

Graphene, a monoatomic layer of $s p^{2}$ bonded carbon, poses the desired properties of ultralarge specific surface area, excellent electrical conductivity [27] and remarkable mechanical flexibility [28,29] for electrochemical supercapacitors. Electrochemical performance of conductive polymers, in term of capacitance values and cycle stability, is particularly enhanced by utilizing graphene and reduced graphene oxide (RGO) as conductive supports [24,30,31]. However, most research efforts in this area deal with discrete 2D graphene sheets composited with PPy [22,24,31], which cannot fully utilize the large surface area and high conductivity of graphene because of the ineffective stacking and disconnection of $2 \mathrm{D}$ graphene sheets. One of the most attractive and easily implementable processes to efficiently realize electrochemical properties of graphene and loaded active materials is to develop a 3D interconnected graphene network. Improvement in supercapacitor performance has been achieved in $\mathrm{Ni}(\mathrm{OH})_{2} / 3 \mathrm{D}$ interconnected graphite network composites [32] and 3D graphene/PPy foams [33]. In this study we report the synthesis and supercapacitor performance of a novel 3D bicontinuous nanotubular graphene-polypyrrole (nt-GPPy) hybrid material with the significant improvement in energy storage of PPy. The nt-GPPy based supercapacitor shows high rate capability and specific capacitance while maintaining structure stability and geometrical integrity of the tubular nano architecture after long-time cycling. 


\section{Experimental procedure}

\subsection{Fabrication of nanotubular graphene}

Nanotubular graphene was prepared by a nanoporous nickel based CVD approach, which has been detailed elsewhere [34]. Briefly, nanoporous nickel (np-Ni) with a pore size of $\sim 10 \mathrm{~nm}$, fabricated by dealloying $150 \mu \mathrm{m}$ thick $\mathrm{Ni}_{30} \mathrm{Mn}_{70}$ alloy sheets, was used as the templates for CVD synthesis of graphene at $800{ }^{\circ} \mathrm{C}$. Graphene continuously grew on ligament surface of np-Ni during the CVD process until the entire surface of ligaments covered by graphene and finally formed a Ni-graphene core-shell structure (graphene@np-Ni) with narrow pore size distribution and average ligament size of 380 nm. Nanotubular graphene@np-Ni hybrid was used as the template to deposit polypyrrole (PPy) on the outer side of the nanotubular graphene.

\subsection{Fabrication of nanotubular graphene-PPy composite}

The synthesis procedure of nanotubular graphene-PPy composite (nt-GPPy) is summarized in Figure 1. Nanoporous Ni-graphene-PPy (np-NiG/PPy) composite was constructed by electrochemically depositing polypyrrole into the graphene@np-Ni using a three-electrode system (Iviumstat electrochemical analyzer, Ivium Technology) with graphene@np-Ni as working electrode and platinum foil and $\mathrm{Ag} / \mathrm{AgCl}$ as counter and reference electrodes, respectively.

Electrochemical deposition was performed using a cyclic voltametry method at a potential window of -0.5 to $0.9 \mathrm{~V}$ and a constant scan rate of $20 \mathrm{mV} \mathrm{s}^{-1}$ in an aqueous electrolyte containing $0.1 \mathrm{M}$ pyrrole monomer and $0.2 \mathrm{M}$ sodium-potassium hydrant tartrate [35]. The graphene@np-Ni template was uniformly coated with nanometer thick PPy and the loading amount of PPy in the hybrid structure was tuned by number of cyclic voltametry electrodepositing. The relationship of the deposition cycle with the mass and thickness of PPy and PPy/graphene ratios are listed in Table S1. The large curvature gradient of graphene results 
in a high density of topological lattice defects, which obviously enhance the wetting ability for easy deposition of PPy coatings [34, 36]. Furthermore, since the pyrrole monomer is a hydrophobic solute in aqueous solutions, weakly hydrated pyrrole monomer solutes in hydrophilic aqueous solution are segregated from the solution and concentrated on hydrophobic surface [37] and hence a PPy layer can be formed as a continuous uniform coating on the graphene surface.

After deposition, the resulting composite was washed with deionized water to remove impurity and excessive pyrrole monomer residue inside pore channels. Finally, 3D interconnected hollow nanotubular microstructure of nt-GPPy composite was achieved by the dissolution of $\mathrm{Ni}$ in $3 \mathrm{M}$ $\mathrm{HCl}$ acid solution. For comparison, we also prepared nanotubular PPy (nt-PPy) in similar way for nt-GPPy by using np-Ni template (annealed at $800^{\circ} \mathrm{C}$ ) instead of graphene@np-Ni template as working electrode during electrodeposition of PPy. The free-standing nt-PPy was obtained by dissolving the nanoporous Ni template.

\subsection{Microstructure characterization and electrochemical measurements}

The microstructures were characterized by JEOL JIB-4600F Scanning Electron Microscopy (SEM), Scaning Electron Microscopy (SEM), JEOL JEM-2100F Transmission Electron Microscopy (TEM) and Raman spectroscopy (Renishaw with an excitation wavelength of $514 \mathrm{~nm}$ and laser power of $10 \mathrm{~mW}$ ). Electrochemical performances of nt-GPPy were evaluated based on a three electrode configuration using Iviumstat electrochemical analyzer, Ivium Technology. The setup was assembled using a platinum sheet as the counter electrode, $\mathrm{Ag} / \mathrm{AgCl}$ as the reference electrode, and nt-GPPy composite as the working electrode. Cyclic voltammograms and galvanostatic charge - discharge curves were recorded in $1 \mathrm{M} \mathrm{HClO}_{4}$ electrolyte at different scan rates and current densities, respectively. Electrochemical impedance spectroscopy (EIS) 
measurements were carried out with the frequency range from $100 \mathrm{kHz}$ to 0.1 with potential amplitude of $10 \mathrm{mV}$.

\subsection{Fabrication and characterization of all solid state supercapacitors}

To fabricate flexible solid state supercapacitore, nt-GPPy composite and polyvinyl alcohol (PVA)- $\mathrm{H}_{2} \mathrm{SO}_{4}$ hydrogel were used as the electrode and electrolyte, respectively. The hydrogel electrolyte was prepared by mixing of $1 \mathrm{~g} \mathrm{H}_{2} \mathrm{SO}_{4}, 1 \mathrm{~g}$ PVA and $10 \mathrm{ml}$ deionized water followed by stirring at $85^{\circ} \mathrm{C}$ until the solution become clear. Then, nt-GPPy composite attached on PET

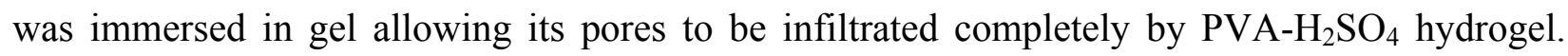
After that, two pieces of nt-GPPy filled by gel were combined together as a symmetric twoelectrode device with a thin sheet of pre-dried hydrogel as both separator and electrolyte sandwiched between them. Finally, the device was dried in a vacuum hood at room temperature for overnight to remove excessive water from the supercapacitor.

\section{Results and discussion}

\subsection{Preparation and characterization of nt-GPPy}

Figure 1 schematically shows the fabrication process of free-standing 3D nt-GPPy composite. The 3D nanotubular graphene was synthesized by chemical vapor deposition using dealloyed nanoporous nickel (np-Ni) as the template. The internal and external surface of np-Ni is entirely coated by an interconnected graphene sheet to form a 3D core-shell graphene@np-Ni [34,38,39]. PPy was loaded on the graphene surface of graphene@np-Ni by cyclic electrochemical deposition [25]. 3D nanotubular graphene-PPy composites (hereafter denoted as nt-GPPy1 to nt-GPPy22 in which the number represents the deposition cycles) were prepared by dissolving the Ni templates in a $\mathrm{HCl}$ solution. The mass and thickness of PPy were controlled by adjusting cycle number during electrochemical deposition process. For comparison, we also prepared nt-PPy without graphene by directly using np-Ni as the template and the resultant 
samples are denoted as nt-PPy1 to nt-PPy10 in which the number represents the deposition cycles. Figures 2a-d show the representative SEM images of the graphene@np-Ni template, nt-GPPy5 and nt-GPPy10 and nt-PPy10. All nt-GPPy samples have a well-defined 3D tubular structure in the form of seamless interconnected network of randomly oriented open nanotube struts. The diameters of the PPy/graphene tubes range from 200 to $600 \mathrm{~nm}$. The morphology of the nonporous graphene@np-Ni template shown in Figure 2a is efficiently duplicated by the 3D architecture of nt-GPPy. Neither detectable shrinkage nor pore collapse and exfoliation of PPy from graphene can be observed after Ni dissolution (Figure 2b and c, and Figure S1), suggesting a strong and robust contact between graphene and PPy. Careful SEM observations from the cross section of the samples reveal that graphene has been thoroughly covered by PPy in whole thickness (Figure S2). BET surface area analysis demonstrates that the nt-GPPy10 composite has a gravimetric surface area of $173 \mathrm{~m}^{2} \mathrm{~g}^{-1}$. The gravimetric surface area increases to $258 \mathrm{~m}^{2} \mathrm{~g}^{-1}$ for nt-GPPy7 and can be further enlarged by decreasing deposition cycles. Both nt-GPPy7 and ntGPPy10 have a very close volumetric surface area of $\sim 38 \mathrm{~m}^{2} \mathrm{~cm}^{-3}$ from both sides of the tubular walls, which is close to the $\mathrm{np}-\mathrm{Ni}$ template $\left(\sim 20 \mathrm{~m}^{2} \mathrm{~cm}^{-3}\right.$ for one side). The consistence in volumetric surface areas between nt-GPPy and np-Ni further demonstrates that the volume change and shrinkage of the nt-GPPy materials is ignorable during the dissolution of np-Ni templates.

Figure 2e displays Raman spectra of nanotubular graphene (nt-G), nt-PPy and nt-GPPy with different PPy deposition cycles. The Raman spectrum of nt-G shows $\mathrm{I}_{2 \mathrm{D}} / \mathrm{I}_{\mathrm{G}}$ and $\mathrm{I}_{\mathrm{D}} / \mathrm{I}_{\mathrm{G}}$ ratios of $\sim 1.77$ and 0.1 , respectively, demonstrating that the nanotubular graphene is mainly comprised of few layer graphene in the form of a continuously interconnected network. The Raman spectrum of nt-PPy is also provided as reference for comparison with nt-GPPy. The main peaks of PPy are located at 1590,1421,1340, 1048, 990 and $929 \mathrm{~cm}^{-1}$. Weak graphene peaks can be 
identified in the spectra of nt-GPPy with PPy plating for 1 and 2 cycles and then disappear in the samples with thicker PPy coatings. Two peaks at 929 and $990 \mathrm{~cm}^{-1}$, which are assigned to C-H out of plane and ring deformation modes and associated with dication (dipolaron) and radical cation (polaron) [40,41], become more intense with deposition cycles. Although, it is difficult to directly measure the doping level in the deposited PPy by the $514 \mathrm{~nm}$ excitation laser, the intensity changes of the two peaks imply the enhanced doping level by increasing number of deposition cycles and thicknesses [41].

Figure 2f indicates the mass of loaded PPy in different deposition cycles. The loading amounts, directly determined by weight measurements, show a nearly linear increase from 1 to 8 cycles. After that, up to 22 cycles, the loading amount with deposition cycles shows a low increasing rate. Similarly, the thickness increases from $\sim 10 \mathrm{~nm}$ in nt-GPPy5 to $\sim 30 \mathrm{~nm}$ in ntGPPy10 while the thickness increment rate becomes low after 10 cycles (Table S1). Mechanism behind this phenomenon can be likely explained by the decrease in nucleation sites of new PPy particles because surface and specialty defects in graphene are expected to provide favorable sites for PPy nucleation [24]. At early deposition cycles, both nucleation and growth of PPy particles seem to contribute to the deposited mass whereas PPy loading amount can be solely from PPy growth after the graphene surface are fully covered.

Figure 3 presents the scanning transmission electron microscopy (STEM) image of the ntGPPy5, which reveals a well-defined bicontinious network with pore sizes of $200-600 \mathrm{~nm}$, similar to SEM images. In the zoom-in STEM image shown in Figure $\mathbf{3 b}$, the surface of graphene is uniformly coated by PPy with a strongly bonded interface. Such kind of interfacial structure not only enhances load transfer capability for improved mechanical properties, but also effectively minimizes electrical resistance at interface for highly conductive composite. Thus, the free-standing hybrid composite is efficiently mimetic outstanding high conductivity of graphene 
and can be used in electrochemical devices, such as supercapacitor, where neither binder nor heavy current collector is required to overcome less integrity and conductivity of active PPy.

\subsection{Electrochemical performance of nt-GPPy in aqueous electrolyte}

The electrochemical capacitance performances of nt-GPPy and nt-PPy were studied by using a three electrode configuration in $1 \mathrm{M} \mathrm{HClO}_{4}$ aqueous electrolyte versus $\mathrm{Ag} / \mathrm{AgCl}$ reference electrode. Figure 4a shows typical cyclic voltammograms (CV) of nt-G, nt-GPPy5, nt-GPPy10 and nt-PPy10 at the scan rate of $50 \mathrm{mV} \mathrm{s}^{-1}$ and the potential windows of -0.1 to $0.6 \mathrm{~V}$. The graphene reinforced PPy samples exhibit superior capacitance performance in comparison with the pure PPy. As shown in Figure S3, the current density of the CV curves increases with scan rates from 10 to $100 \mathrm{mV} \mathrm{s}^{-1}$. The $\mathrm{CV}$ curves of the nt-GPPy5 electrode have a symmetric and nearly rectangular shape at all scan rates, demonstrating highly reversible charge and discharge with excellent rate capability [8,9]. It is also evident that the current-voltage response shows no obvious redox peaks in the recorded potential window from -0.1 to $0.6 \mathrm{~V}$ during both positive and negative sweeps, indicating that the electrode is charged and discharged at a pseudoconstant rate over the entire CV process $[22,42,43]$. The Nyquist plots of nt-GPPy5, nt-GPPy10 and nt-PPy10 obtained by using electrochemical impedance spectroscopy (EIS) in the frequency range from $100 \mathrm{kHz}$ to $0.1 \mathrm{~Hz}$ are shown in Figure 4b. EIS data evaluate the frequency dependence of electrochemical properties [17]. Ion diffusion resistance at electrode/electrolyte interface and proton transport in host electrodes are generally explained by the deviation from vertical part of frequency dependence of EIS. The Nyquist plots of all nt-GPPy electrodes have a near straight line in the low frequency region, indicating low ion/electrolyte diffusion resistance, approaching ideal capacitance compared to nt-PPy. The semicircles in high frequency region are attributed to the electrical charge transfer and faradic resistance at electrodes and electrode/collector interfaces. The charge transfer resistance of nt-PPy10 is $\sim 70 \Omega$ and reduces to $35 \Omega$ for nt-GPPy22, 
suggesting a lower electrode resistance and higher charge-transfer rate in the nt-GPPy electrodes. The smaller radius of the semicircles in the high frequency range for the nt-GPPy5 and ntGPPy10 indicates that the low charge transfer resistance mainly arises from the loaded PPy and thinner PPy shells give lower charge transfer resistance.

The electrochemical properties of the electrodes were also characterized by galvanostatic charge/discharge measurements (Figure 4c). The nt-GPPy electrodes exhibit superior performance than pristine nt-PPy, further demonstrating the importance of reinforcement from the nanotubular graphene. Notably, the small voltage drop at the beginning of discharge stage together with slow voltage loss in respect to discharge time at nt-GPPy suggests very low internal resistance, which can be ascribed to the excellent electrical conductivity of the $3 \mathrm{D}$ interconnected graphene network and the large contact area between the graphene and active PPy. Excellent conductivity of continuously interconnected nanoporous graphene can be well retained in the composite. Hybrid composite of nt-GPPy5 showed high electrical conductivity of $863 \mathrm{~S} \mathrm{~m}^{-1}$ as measured by four probe method. Specific capacitance was calculated from discharge curves by using following equation: $S C=I / m(\Delta \mathrm{V} / \Delta \mathrm{t})$

where $\boldsymbol{S C}$ is the specific capacitance, $I$ is the discharge current, $m$ is the mass of loaded PPy, and $\Delta V / \Delta t$ is the slop of discharge curve after voltage drop [8,9]. Figure 4d summarizes the rate dependence of the specific capacitance of nt-GPPy with different deposition cycles in comparison with the pristine nt-PPy. The specific capacitance decreases gradually with increasing current density because the time constraint of diffusion limits the movement of ions at high current density $[42,43]$ and the utilization of accessible active surface for charge storage. The high specific capacitance of $\sim 457 \mathrm{~F} \mathrm{~g}^{-1}$ could be delivered by nt-GPPy10 at $0.15 \mathrm{~A} \mathrm{~g}^{-1}$, which is much higher than that of nt-PPy10 $\left(\sim 300 \mathrm{~F} \mathrm{~g}^{-1}\right)$ with identical mass loading of PPy $\left(\sim 170 \mathrm{mg} \mathrm{cm}^{-3}\right)$. As the current density increases from 0.15 to $3 \mathrm{~A} \mathrm{~g}^{-1}$, the specific capacitance of the pristine nt- 
PPy 10 drops to almost $20 \%$ of the initial capacitance. In contrast, nt-GPPy10 maintains $55 \%$ of initial capacitance, demonstrating a much better rate performance of nt-GPPy. Although the good rate dependence could be preserved for the highest amount of PPy loading (nt-GPPy22), the ntGPPy22 has much lower specific capacitance of $280 \mathrm{~F} \mathrm{~g}^{-1}$ at the current density of $0.15 \mathrm{~A} \mathrm{~g}^{-1}$, which is associated with the loss of specific surface area of thick PPy coatings and the increment in charge transfer resistance [24] as the doping/undoping process for charge storage mainly takes place on surfaces. Additionally, the contribution from graphene in the charge storage by the electrochemical double layer effect is minor in comparison with the pseudocapactive PPy (Figure 4a). Among all the nt-GPPy samples, the nt-GPPy5 shows the best combination of high rate performance of $65 \%$ as the current density increased to as high as $3 \mathrm{~A} \mathrm{~g}^{-1}$ together with high specific capacitance of $509 \mathrm{~F} \mathrm{~g}^{-1}$ at $0.15 \mathrm{~A} \mathrm{~g}^{-1}$, which is much higher than PPy aerogel on carbon fiber cloth (380 F g $\mathrm{g}^{-1}$ at $\left.0.2 \mathrm{~A} \mathrm{~g}^{-1}\right)$ and electrochemically deposited PPy on graphene or reduced graphene oxide $[24,44,45]$. Intriguingly, the specific capacitance calculated on the basis of the total weight of electrode (including the graphene supports) is still as high as 352 and $366 \mathrm{~F} \mathrm{~g}^{-1}$ for nt-GPPy5 and 10, respectively (Figure S3d), which is also much higher than reported graphene/PPy [45], graphene/CNT/PPy and even metal oxide based electrode like graphite foam/ $\mathrm{Ni}(\mathrm{OH})_{2}$ [32] and $\mathrm{CNT} / \mathrm{MnO}_{2} / \mathrm{PPy}$ [46]. The high capacitance of the nt-GPPy hybrid electrode can be ascribed to the synergistic effect between graphene and PPy. 3D interconnected nanotube with open porosity offers a large accessible surface area for high charge storage and fast transport of electrolyte ions as well as charge transfer reactions. The 3D graphene network in the composites not only buffers the volume change during the charging and discharging process, but also preserves the high electrical conductivity of the entire electrode thanks to the excellent conductivity of graphene. 
Conducting polymers as supercapacitor electrodes usually suffer from poor cycling stability because the swelling and shrinking of the polymers may lead to degradation, which restricts the low-cost electrode materials for commercial applications. The cycling stability of ntGPPy5, nt-GPPy10 and nt-PPy10 were examined by using a CV method at a scan rate of $50 \mathrm{mV}$ $\mathrm{s}^{-1}$, as shown in Figure 5. Excellent cycle stability for both nt-GPPy and nt-PPy can be seen as the capacitance retention of nt-PPy10, nt-GPPy5 and nt-GPPy10 after 1500 cycles are $91.6 \%$, $93 \%, 94.5 \%$ respectively. Cycling test of nt-GPPy 10 was extended to 13000 cycles at which the capacitance decreases to $75 \%$ of initial value (Figure $5 \mathbf{b}$ ). The excellent durability at a high specific capacitance suggests that the nt-GPPy hybrid materials are a suitable candidate for electrochemical supercapacitor applications. SEM and Raman characterization were conducted to investigate the microstructure changes caused by $\mathrm{CV}$ cycling. By comparing the images of ntGPPy5 and nt-GPPy10 before (Figure 2b,c) and after cycling (Figure S4a,b) (1500 cycles for ntGPPy5 and 13200 cycles for nt-GPPy10), obvious shrinkage cannot be seen as the thickness of sample is $112 \mu \mathrm{m}$, very close to the original value of $115 \mu \mathrm{m}$. Additionally, the interconnected nanotubular network is well retained (inset SEM images in Figure S4a,b) without any pore collapse, suggesting that the continuous electrically conductive pathway remains invariant. From Raman data in Figure S4c, we cannot found any significant differences in characteristic peaks of PPy, demonstrating the absence of irreversible structure change originated from electrochemical cycling. However, a small intensity loss at peaks of 1048 and 929 in nt-GPPy10 can be detected after 13200 cycles, which may be associated with the decreased thickness of PPy [25,41] after long time cycling. 


\subsection{Solid-state supercapacitor}

Because the nanotubular graphene/PPy composites are mechanically flexible, we fabricated all solid state symmetric supercapacitors using nt-GPPy as electrodes and a $\mathrm{PVA} / \mathrm{H}_{2} \mathrm{SO}_{4}$ gel as electrolyte for flexible energy storage devices. The capacitive performance of the flexible symmetric solid state supercapacitors was evaluated by $\mathrm{CV}$ and galvanostatic charge/discharge tests. The CV curves of nt-GPPy5 and nt-GPPy10 at scan rates ranging from 2 to $100 \mathrm{mV} \mathrm{s}^{-1}$ are illustrated in Figure S5, showing a very rapid current response on the voltage reversal at each end potential for both samples. The rectangular shape of the CV curves can be kept even at a high scan rate of $100 \mathrm{mV} \mathrm{s}^{-1}$, indicating a small ESR and faster ionic diffusion of the all solid state electrodes. Figure 6a displays charge-discharge curves of nt-GPPy5 based supercapacitor at different current densities of $0.2 \mathrm{~A} \mathrm{~g}^{-1}$ to $20 \mathrm{~A} \mathrm{~g}^{-1}$. Identical to $\mathrm{CV}$ measurements, a symmetric triangular shape of the charge-discharge profiles further confirms the formation of a nearly ideal capacitor with efficient charge propagation across two symmetric electrodes. Figure 6b summarizes the specific capacitance of nt-GPPy5 and nt-GPPy10 at different current densities calculated from discharge curves. The specific capacitances of the ntGPPy5 and nt-GPPy10 electrodes are 514 and $512 \mathrm{~F} \mathrm{~g}^{-1}$ at the current density of $0.2 \mathrm{~A} \mathrm{~g}^{-1}$, and decrease to 420 and $370 \mathrm{~F} \mathrm{~g}^{-1}$ at the current density of $1 \mathrm{~A} \mathrm{~g} \mathrm{~g}^{-1}$, which are noticeably higher than those of all symmetric supercapacitore in aqueous electrolytes, including 3D graphene foam/PPy (350 $\mathrm{F} \mathrm{g}^{-1}$ at $1.5 \mathrm{~A} \mathrm{~g}^{-1}$ ) [33], MWCNT/PPy/RGO/NWF (320 $\mathrm{F} \mathrm{g}^{-1}$ at $1 \mathrm{mV} \mathrm{s}^{-1}$ ) [47] and RGO/PPy (284 $\mathrm{F} \mathrm{g}^{-1}$ at $10 \mathrm{mV} \mathrm{s}^{-1}$ ) [22]. The nt-GPPy5 shows lower capacitance fading and better rate performance than nt-GPPy10. When current density is increased by 100 fold (to $20 \mathrm{~A} \mathrm{~g}^{-1}$ ), the specific capacitance has $63 \%$ retention. Excellent capacitance performance and rate capability, comparable to the aqueous ones, suggest that the $3 \mathrm{D}$ open porous nanotubular composite is efficiently impregnated by the gel electrolyte, leading to the enhanced contact interface between 
PPy and electrolyte. Effective filling of the polymer electrolyte into the nanotubular microstructure was also confirmed as no obvious change was observed when the cell was bended (Figure S5c). Furthermore, both nt-GPPy5 and nt-GPPy10 exhibit good cycling stability with almost 95\% retention of initial state after 1000 cycles as shown in Figure 6c. To further highlight the superior performances of the all solid-state nt-GPPy based supercapacitors, the supercapacitor performances are compared with other graphene-PPy composites in the Ragone plot (Figure 6d). Energy density $(E)$ and power density $(P)$ were determined by $E=0.5 C V^{2}$ and $P=V^{2} / 4 R m$, where $\boldsymbol{C}$ and $\boldsymbol{V}$ are the cell capacitance and cell voltage, respectively; $\boldsymbol{m}$ is the mass of PPy in both electrodes; and $\boldsymbol{R}$ is internal resistance [8]. From the plot of specific power density versus energy density we can found that the nt-GPPy based supercapacitors possess exceptional combination of high power and energy densities, which are shown in upper right portion of the Ragone plot. The reported data from the best PPy based supercapacitors from literature are also summarized in the plot for comparison. Inspection of Figure 6d elucidates that nt-GPPy10 has the power density up to $3.36 \mathrm{~kW} \mathrm{~kg}^{-1}$ at the energy density of $12 \mathrm{Wh} \mathrm{kg}^{-1}$ or higher power of 12 $\mathrm{kW} \mathrm{kg}^{-1}$ at energy density of $4.7 \mathrm{Wh} \mathrm{kg}^{-1}$, which are comparable to recently reported SWCNT/PPy/ $\mathrm{MnO}_{2}$ [46] complex electrode, but has better flexibility and stability. By reducing the PPy loading amount, the nt-GPPy5 based supercapacitor can offer the ultrahigh power density and energy density of $60.1 \mathrm{~kW} \mathrm{~kg}^{-1}$ and $21.6 \mathrm{Wh} \mathrm{kg}^{-1}$ even better than PPy@3D-Ni [48]. Therefore, the supercapacitor performance of the nt-GPPy electrodes, in the term of specific capacitance, rate performance, lifetimes and energy/power densities, are much higher than most of previously reported devices fabricated by graphene/PPy [49], graphene oxide(GO)/PPy [50,51], reduced graphene oxide/PPy [52] and graphene oxide /PPy on Ni foam [53]. Consequently, in addition to light weight and mechanical flexibility, such unprecedented supercapacitor properties set the novel composite electrodes apart from all graphene/PPy based supercapacitors. 


\section{Conclusion}

In summary we have successfully developed a new 3D interconnected nanotubular graphene-polypyrrole hybrid material by electrodepositing polypyrrole on CVD synthesized nanotubular graphene. The nanotubular hybrid composites used as free standing electrodes in supercapacitor show high specific capacitance of $509 \mathrm{~F} \mathrm{~g}^{-1}$, excellent cycle stability and outstanding rate performance. Moreover, all-solid-state flexible supercapacitor fabricated by the hybrid material and $\mathrm{PVA} / \mathrm{H}_{2} \mathrm{SO}_{4}$ gel electrolyte shows the specific capacitance as high as $514 \mathrm{~F} \mathrm{~g}^{-}$

${ }^{1}$ at a discharge current density of $0.2 \mathrm{~A} \mathrm{~g}^{-1}$ and the maximum energy density of $21.6 \mathrm{Wh} \mathrm{kg}^{-1}$ at power density of $32.7 \mathrm{~kW} \mathrm{~kg}^{-1}$, among the highest values reported for PPy-carbon based supercapacitors. The outstanding supercapacitor performance of the nanotubular graphenepolypyrrole material benefits from the high electric conductivity and excellent electrochemical and mechanical stability of the nanoporous graphene support which has unique bicontinuous open porosity. This study may open up the possibility to realize fundamental properties of conducting polymers into technologically viable light weight and flexible energy devices with high energy and power densities as well as long lifetimes.

\section{Acknowledgements}

This work is sponsored by JST-CREST "Phase Interface Science for Highly Efficient Energy Utilization", JST, Japan; and World Premier International (WPI) Research Center Initiative for Atoms, Molecules and Materials, MEXT, Japan.

\section{Appendix A. Supporting information}

Supplementary data associated with this article can be found in the online version. 


\section{References}

[1] P. Simon, Y. Gogotsi, Nat. Mater. 7 (2008) 845-854.

[2] P. Simon, Y. Gogotsi, B. Dunn, Science 243 (2014) 1210-1211.

[3] Y. Zhu, S. Murali, M.D. Stoller, K.J. Ganesh, W. Cai, P.J. Ferreira, A. Pirkle, R.M. Wallace, K.A. Cychosz, M. Thommes, D. Su, E.A. Stach, R.S. Ruoff, Science 332 (2011) 1537-1541.

[4] J.H. Han, Y.C. Lin, L.Y. Chen, Y.C. Tsai, Y. Ito, X.W.Guo, A. Hirata, T. Fujita, M.Esashi, T.Gessner, M.W. Chen, Adv. Sci. 2 (2015) 1500067-1-7.

[5] Y. Tao, X. Y. Xie, W. Lv , D. M. Tang, D. B. Kong, Z. H. Huang, H. Nishihara, T. Ishii, B. H. Li, D. Golberg, F. Y. Kang, T. Kyotani, Q. H. Yang, Sci. Rep. 3 (2013) 2975-1-8.

[6] Y. Yang, L. Li, G. Ruan, H. Fei, C. Xiang, X. Fan, J.M. Tour, ACS Nano 8 (2014) 9622-9628.

[7] J.P. Liu, J. Jiang, C.W. Cheng, H.X. Li, J.X. Zhang, H. Gong, H.J. Fan, Adv. Mater. 23 (2011) 2076-2081.

[8] L.Y. Chen, Y. Hou, J.L. Kang, A. Hirata, T. Fujita, and M.W. Chen, Adv. Energy Mater. 3 (2013) 851-856.

[9] X.Y. Lang, A. Hirata, T. Fujita, M.W. Chen, Nat. Nanotechnol. 6 (2011) 232-236.

[10] X. Wang, J. Deng, X. Duan, D. Liu, J. Guo, P. Liu, J. Mater. Chem. A 2 (2014) 1232312329.

[11] Y. Zhou, H. Xu, N. Lachman, M. Ghaffari, S. Wu, Y. Liu, A. Ugur, K.K. Gleason, B.L. Wardle, Q. M. Zhang, Nano Energy 9 (2014) 176-185.

[12] J. Yan, Z. Fan, W. Sun, G. Ning, T. Wei, Q. Zhang, R. Zhang, L. Zhi, F. Wei, Adv. Funct. Mater. 22 (2012) 2632-2641.

[13] F. Meng, Y. Ding, Adv. Mater. 23 (2011) 4098-4102. 
[14] J. Zhou, Z. Zhang, W. Xing, J. Yu, G. Han, W. Si, S. Zhuo, Electrochim. Acta 153 (2015) 68-75.

[15] Y. Xu, Z. Lin, X. Huang, Y. Liu, Y. Huang, X.F. Duan, ACS Nano 7 (2013) 4042-4049.

[16] W. Lv, Z. Li, G. Zhou, J.-J. Shao, D. Kong, X. Zheng, B. Li, F. Li, F. Kang, Q.H. Yang Adv. Funct. Mater. 24 (2014) 3456-3463.

[17] X. Zheng, W. Lv, Y. Tao, J. Shao, C. Zhang, D. Liu, , J. Y. Luo, D. W. Wang, Q.H. Yang, Chem. Mater. 26 (2014) 6896-6903

[18] Y. Yang, C. Wang, B. Yue, S. Gambhir, C.O. Too, G.G. Wallace, Adv. Energy Mater. 2 (2012) 266-272.

[19] G.A. Snook, P. Kao, A.S. Best, J. Power Sources 196 (2011) 1-12.

[20] J.H. Kim, Y.S. Lee, A.K. Sharma, C.G. Liu, Electrochim. Acta 52 (2006) 1727-1732.

[21] Y. Hou, L. Zhang, L.Y. Chen, P. Liu, A. Hirata, M.W. Chen, Phys. Chem. Chem. Phys. 16 (2014) 3523-3528.

[22] J. Zhang, P. Chen, B.H.L. Oh, M.B.C. Park, Nanoscale 5 (2013) 9860-9866.

[23] Q. Li, N. Mahmood, J. Zhu, Y. Hou, S. Sun, Nano Today 9 (2014) 668-683.

[24] A. Davies, P. Audette, B. Farrow, F. Hassan, Z. Chen, J.Y. Choi, A. Yu, J. Phys. Chem. C 115 (2011) 17612-17620.

[25] Y. Hou, L. Chen, L. Zhang, J. Kang, T. Fujita, J. Jiang, M.W. Chen, J. Power Sources 225 (2013) 304-310.

[26] J. Wei, G.Z. Xing, L. Gao, H. Suo, X.P. He, C. Zhao, S. Li, S.X. Xing, New J. Chem. 37 (2013) 337-341.

[27] M.T. Pettes, H. Ji, R.S. Ruoff, L. Shi, Nano Lett. 12 (2012) 2959-2964.

[28] C. Lee, X. Wei, J.W. Kysar, J. Hone, Science 321(2008) 385-388. 
[29] G.H. Lee, R.C. Cooper, S.J. An, S. Lee, A. van der Zande, N. Petrone, A.G. Hammerberg, C. Lee, B. Crawford, W. Oliver, J.W. Kysar, Science 340 (2013) 1073-1076.

[30] B. Wang, J. Qiu, H. Feng, E. Sakai, Electrochim. Acta 151 (2015) 230-239.

[31] X.J. Lu, F. Zhang, H. Dou, C.Z. Yuan, S.D. Yang, L. Hao, L.F. Shen, L.J. Zhang, X.G. Zhang, Electrochim. Acta 69 (2012) 160-166.

[32] J. Ji, L.L. Zhang, H. Ji, Y. Li, X. Zhao, X. Bai, X. Fan, F. Zhang, R.S. Ruoff, ACS Nano 7 (2013) 6237-6243.

[33] Y. Zhao, J. Liu, H.H. Chen, C.G. Hu, C.C. Jiang, L. Jiang, A.Y. Cao, L.T. Qu, Adv. Mater. 25 (2013) 591-595.

[34] Y. Ito, Y. Tanabe, H.J. Qiu, K. Sugawara, S. Heguri, N.H. Tu, K.K. Huynh, T. Fujita, T. Takahashi, K. Tanigaki, M.W. Chen, Angew. Chem. Int. Ed. 53 (2014) 4922-4926.

[35] L.M. Martins dos Santos, J.C. Lacroix, K.I. Chane-Ching, A. Adenier, L.M. Abrantes, P.C. Lacaze, J. Electroanal. Chem. 587 (2006) 67-78.

[36] Y. Ito, Y. Tanabe, J.H. Han, T. Fujita, K. Tanigaki, M. W. Chen, Adv. Mater. 27 (2015) 4302-4307.

[37] A. Koyama, K. Fukami, T. Yamauchi, N. Nishi, T. Sakka, A. Kitada, K. Murase, ECS Electrochem. Lett. 3 (2014) G5-G7.

[38] Y. Ito, H.J. Qiu, T. Fujita, Y. Tanabe, K. Tanigaki, M.W. Chen, Adv. Mater. 26 (2014) 4145-4150.

[39] Y. Ito, C. Weitao, T. Fujita, Z. Tang, M.W. Chen, Angew. Chem. Int. Ed. 54 (2015) 21312136.

[40] Y.C. Liu, B.J. Hwang, W.J. Jian, R Santhanam, Thin Solid Films 374 (2000) 85-91.

[41] F. Chen, G. Shi, M.X. Fu, L. Qu, X.Y. Hong, Synthetic Metal 132 (2003) 125-132. 
[42] J. Zhou, Z. Zhang, W. Xing, J. Yu, G. Han, W. Si, S. Zhuo, Electrochim. Acta 153 (2015) $68-75$.

[43] M. Li, Z. Tang, M. Leng, J. Xue, Adv. Funct. Mater. 24 ( 2014) 7495-7502.

[44] Y. Shi, L. Pan, B. Liu, Y. Wang, Y. Cui, Z. Bao, G. Yu, J. Mater. Chem. A 2 ( 2014) 60866091.

[45] H.P. Oliveira, S.A. Sydlik, T.M. Swager. J. Phys. Chem. C 117 (2013) 10270-10276.

[46] K. Liang, T. Gu, Z. Cao, X. Tang, W. Hu, B. Wei, Nano Energy 9 (2014) 245-251.

[47] F. Liu, S. Wang, G. Han, R. Liu, Y. Chang, Y. Xiao, J. Appl. Polym. Sci. 131 (2014) 41023$1-10$.

[48] G.F. Chen, Y.Z. Su, P.Y. Kuang, Z.Q. Liu, D.Y. Chen, X. Wu, N. Li, S.Z. Qiao, Chem. Eur. J. 21 (2015) 4614-4621.

[49] D.C. Zhang, X. Zhang, Y. Chen, P. Yu, C.H. Wang, Y.W. Ma, J. Power Sources 196 (2011) $5990-5996$.

[50] S. Bose, N.H. Kim, T. Kuila, K.T. Lau, J.H. Lee, Nanotechnology 22 (2011) 292502-1-9.

[51] L.Q. Fan, G.J. Liu, J.H. Wu, L. Liu, J.M. Lin, Y.L. Wei, Electrochim. Acta 137 (2014) 2633.

[52] X. Cai, S.H. Lim, C.K. Poh, L.F. Lai, J.Y. Lin, Z. Shen, J. Power Sources 275 (2015) 298304.

[53] W.K. Chee, H.N. Lim, I. Harrison, K.F. Chong, Z. Zainal, C.H. Ng, N.M. Huang, Electrochim. Acta 157 (2015) 88-94. 


\section{Figure captions}

Figure 1 Schematic of preparation steps of nanotubular graphene/PPy composite. (a) nanoporous Ni-graphene hybrid composite (graphene@np-Ni) prepared by chemical vapor deposition. (b) Nanoporous nickel/graphene/PPy prepared by electrodeposition of PPy on the surface of graphene@np-Ni ligaments. (c) Nanotubular-GPPy after removing Ni substrate by chemical etching.

Figure 2 Microstructure characterization of nt-GPPy hybrid electrodes. (a) SEM image of graphene@np-Ni. (b) SEM micrograph of nt-GPPy5. (c) SEM micrograph of nt-GPPy10. (d) Pristine nt-PPy10 after etching Ni. (e) Raman spectra of nt-GPPy and nt-PPy with different deposition cycles. Raman spectra are collected before removing the Ni substrates. (f) Deposition mass of PPy versus deposition cycles. The insets of (b) and (c) are zoom-in SEM images showing the tubular structure.

Figure 3 STEM image of nt-GPPy5. (a) STEM image shows tubular morphology of nt-GPPy5. (b) High magnification of region marked in (a). Inset in (b) is high magnification image of marked in (b) showing multilayers graphene support and bonding with PPy. Scale bar is $0.2 \mu \mathrm{m}$ in (a), and $10 \mathrm{~nm}$ in (b).

Figure 4 Supercapacitance performance of nt-GPPy and nt-PPy in $1 \mathrm{M} \mathrm{HClO}_{4}$ electrolyte. (a) CV curves of nt-G, nt-GPPy5, nt-GPPy10 and nt-PPy10 at scan rate of $50 \mathrm{mV} \mathrm{s}^{-1}$. (b) EIS results of nt-GPPy5, nt-GPPy10, nt-GPPy22 and nt-PPy10. (c) Charge-discharge curves of nt-GPPy5, ntGPPy10 and nt-PPy10 at $0.5 \mathrm{~A} \mathrm{~g}^{-1}$. (d) Specific capacitance versus current density.

Figure 5 Cycling performance of nanotubular electrodes in $1 \mathrm{M} \mathrm{HClO}_{4}$ electrolyte at scan rate of $50 \mathrm{mV} \mathrm{s}^{-1}$. (a) Capacitance retention of nt-PPy10, nt-GPPy5 and nt-GPPy10. Specific 
capacitances of nt-GPPy5, nt-GPPy10 and nt-PPy10 decrease to 91.6, 93 and $94.5 \%$ of their original values after 1500 cycles respectively. (b) Capacitance retention of nt-GPPy10 after 13200 cycles. Specific capacitance is reduced to $75 \%$ after 13200 cycles at $50 \mathrm{mV} \mathrm{s}^{-1}$. Inset shows CV curve of first and $13200^{\text {th }}$ cycle.

Figure 6 Solid state supercapacitance performance of nt-GPPy5 and nt-GPPy10 integrated with PVA- $\mathrm{H}_{2} \mathrm{SO}_{4}$ solid electrolyte. (a) Charge-discharge curves of nt-GPPy5 as a solid state supercapacitor at different current densities. (b) Specific capacitance versus current density of the symmetric solid state supercapacitor assembled by nt-GPPy5 and nt-GPPy10. Inset shows a schematic of the solid state supercapacitor. (c) Capacitance retention of nt-GPPy5 and nt-GPPy10. (d) Power density versus energy density of nt-GPPy5 and nt-GPPy10 and recently reported data from literature. 
(a) Core-shell graphene@np-Ni

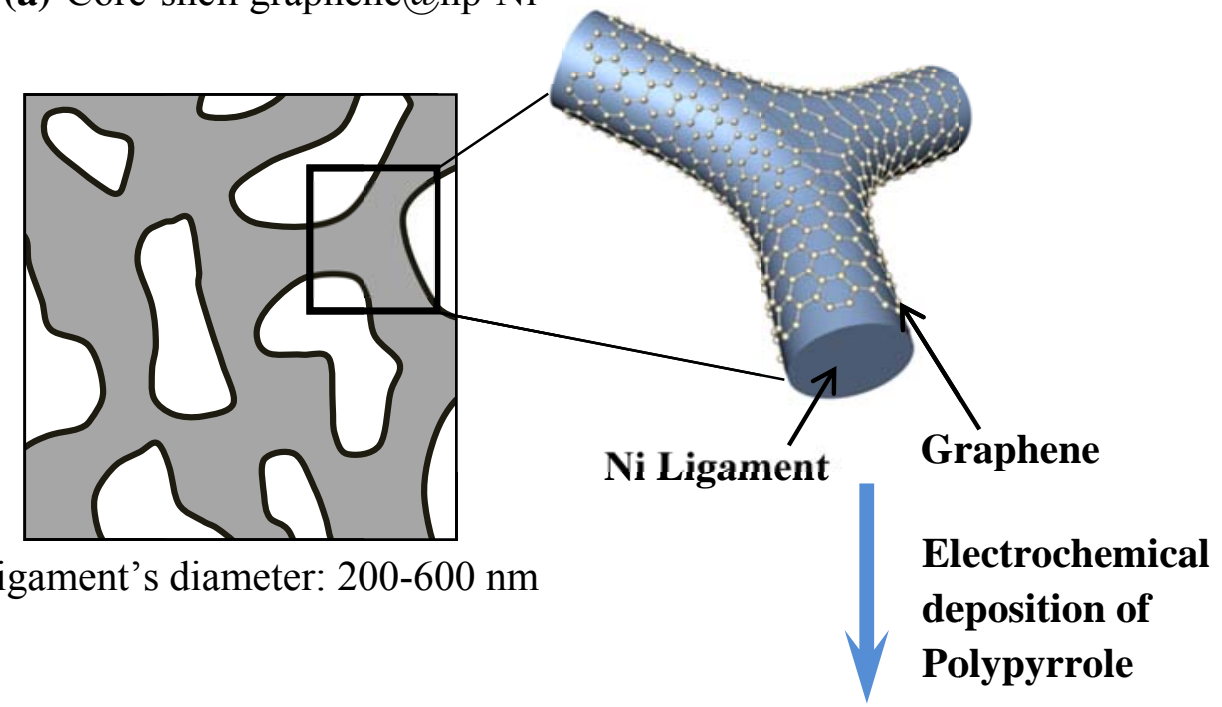

(c) Nanotubular graphenepolypyrrole hybrid

(b) Polypyrrole on surface of
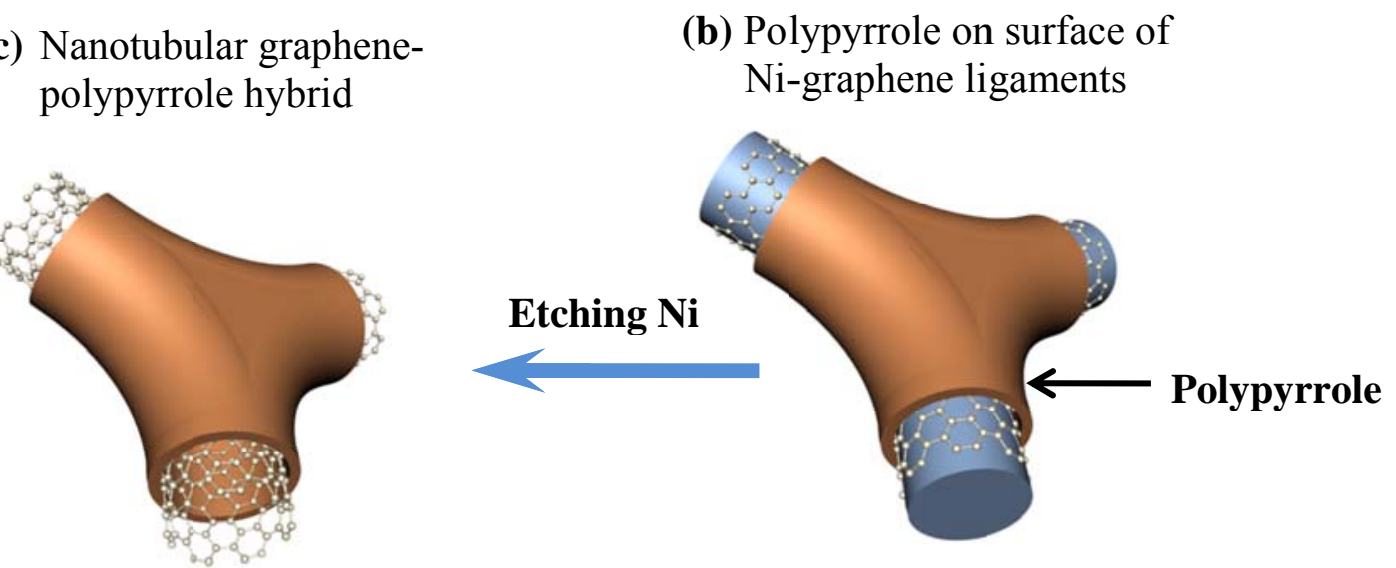

Nanotube's diameter: 200-600 nm

Figure 1 Schematic of preparation steps of nanotubular graphene/PPy composite. (a) Nanoporous Ni-graphene hybrid composite (graphene@np-Ni) prepared by chemical vapor deposition. (b) Nanoporous nickel/graphene/PPy prepared by electrodeposition of PPy on the surface of graphene@np-Ni ligaments. (c) Nanotubular-GPPy after removing Ni substrate by chemical etching. 

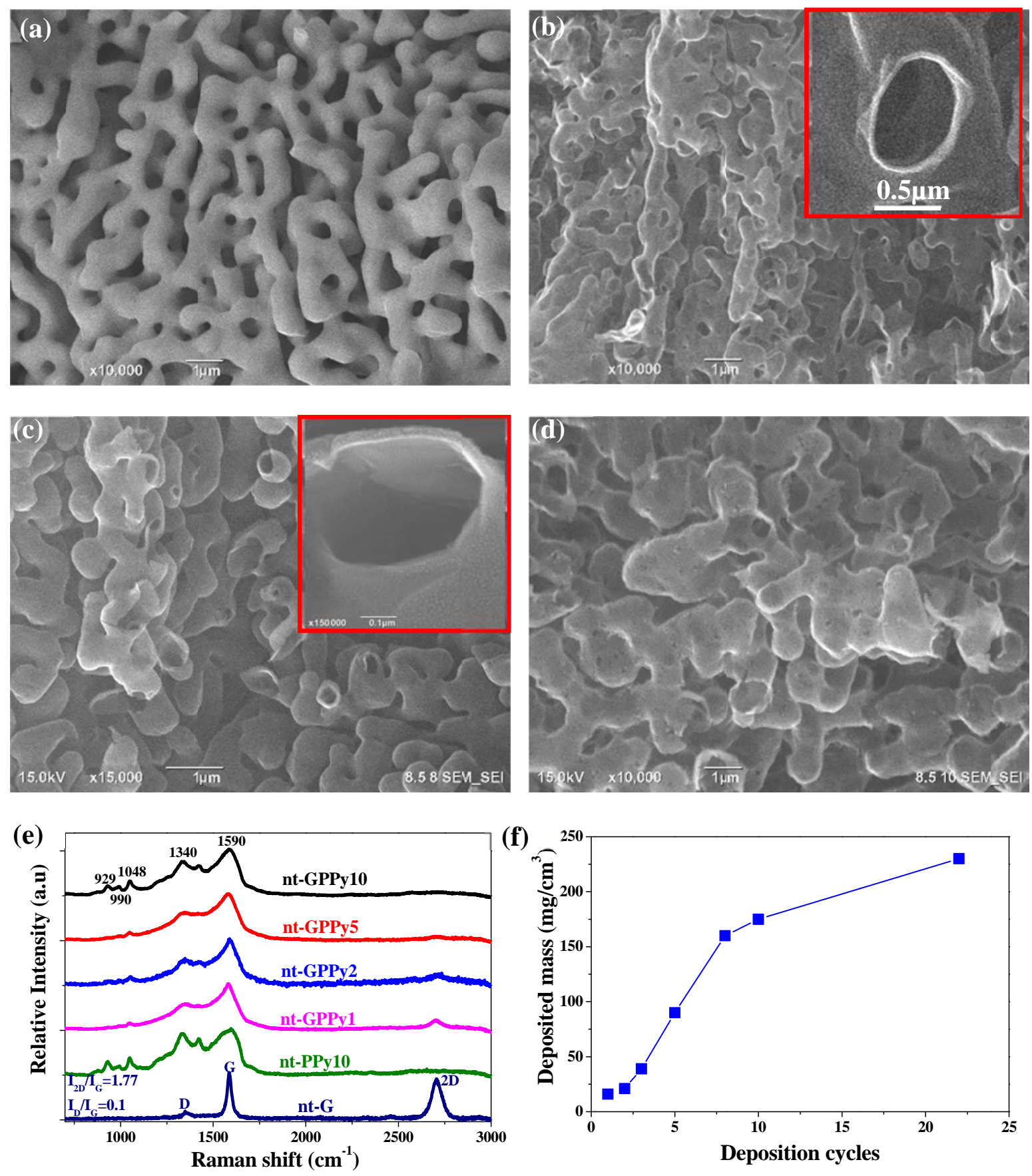

Figure 2 Microstructure characterization of nt-GPPy hybrid electrodes. (a) SEM image of graphene@np-Ni. (b) SEM micrograph of nt-GPPy5. (c) SEM micrograph of nt-GPPy10. (d) Pristine nt-PPy10 after etching Ni. (e) Raman spectra of nt-GPPy and nt-PPy with different deposition cycles. Raman spectra are collected before removing the Ni substrates. e) Deposition mass of PPy versus deposition cycles. The insets of (b) and (c) are zoom-in SEM images showing the tubular structure. 


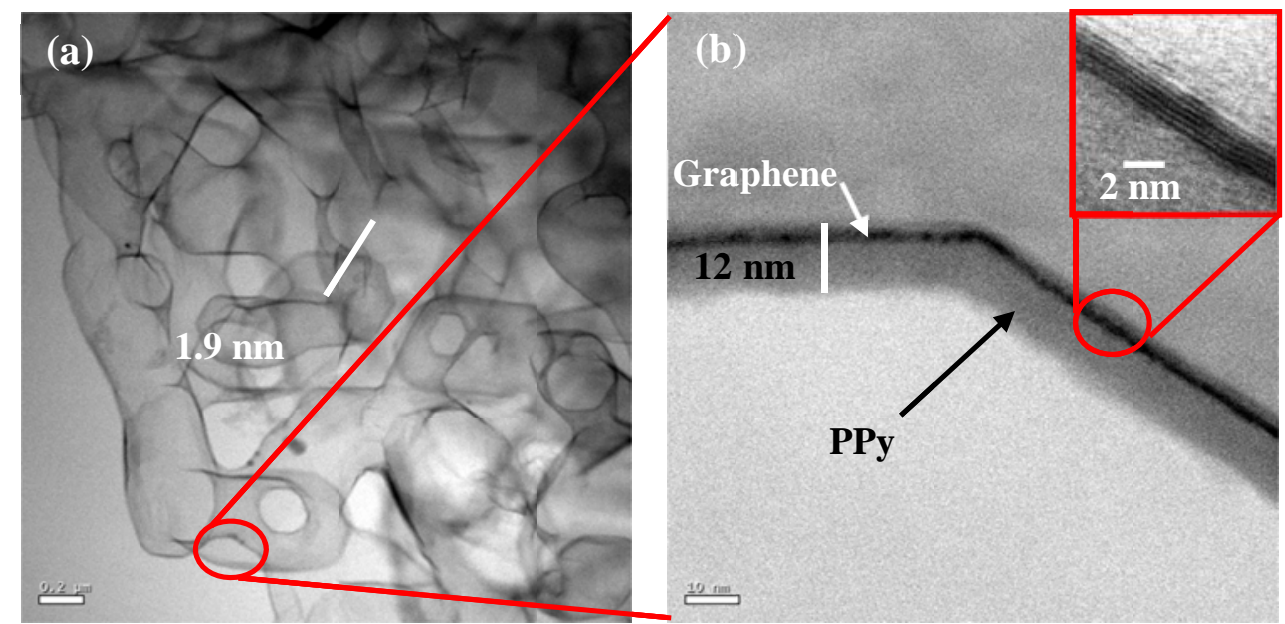

Figure 3 STEM image of nt-GPPy5. (a) STEM image shows tubular morphology of nt-GPPy5. (b) High magnification of region marked in (a). Inset in (b) is high magnification image of marked in (b) showing multilayers graphene support and bonding with PPy. Scale bar is $0.2 \mu \mathrm{m}$ in (a), and $10 \mathrm{~nm}$ in (b). 

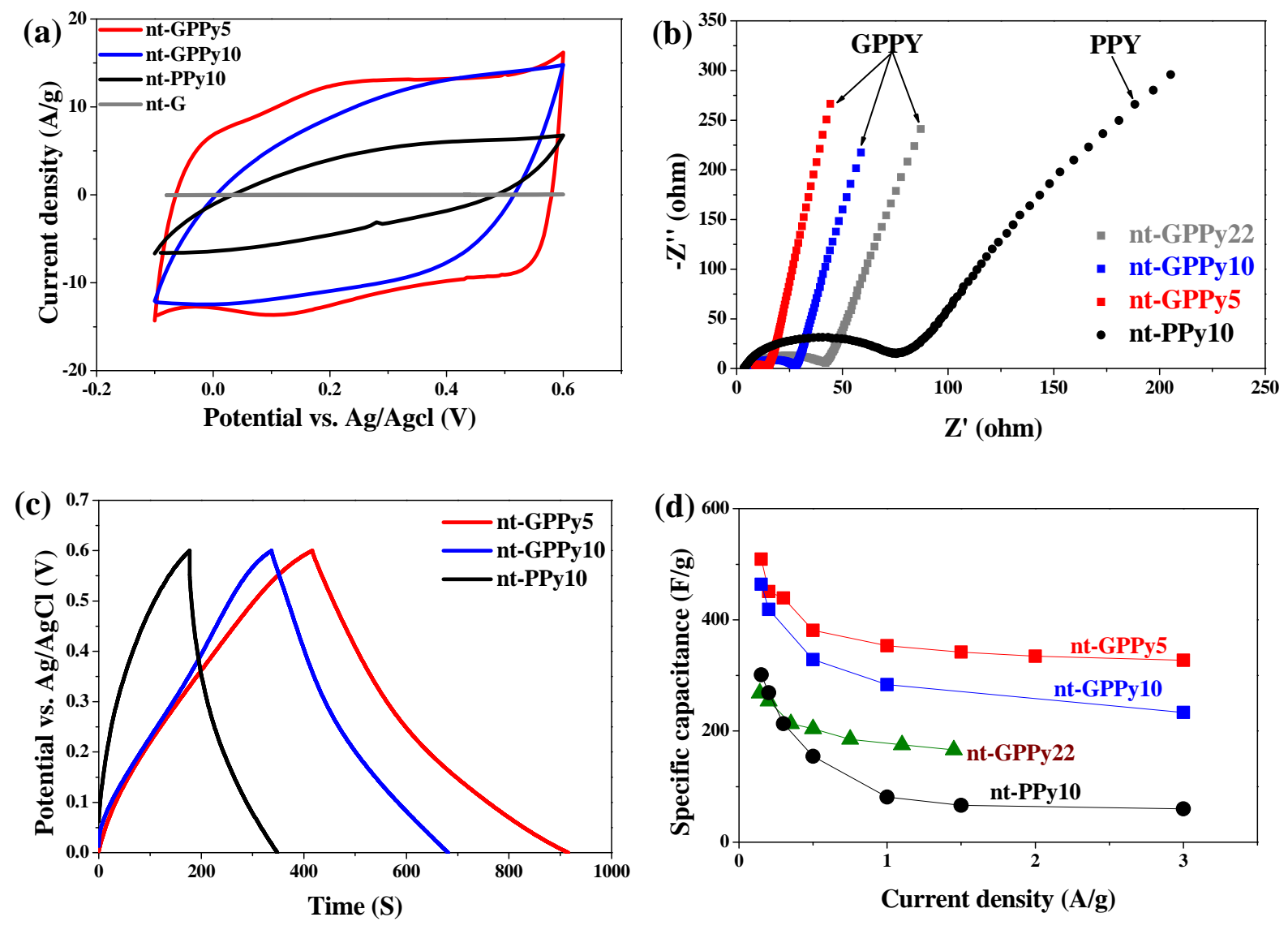

Figure 4 Supercapacitance performance of nt-GPPy and nt-PPy in $1 \mathrm{M} \mathrm{HClO}_{4}$ electrolyte. (a) CV curves of nt-G, nt-GPPy5, nt-GPPy10 and nt-PPy10 at scan rate of $50 \mathrm{mV} \mathrm{s}^{-1}$. (b) EIS results of nt-GPPy5, nt-GPPy10, nt-GPPy22 and nt-PPy10. (c) Charge-discharge curves of nt-GPPy5, ntGPPy10 and nt-PPy10 at $0.5 \mathrm{~A} \mathrm{~g}^{-1}$. (d) Specific capacitance versus current density. 

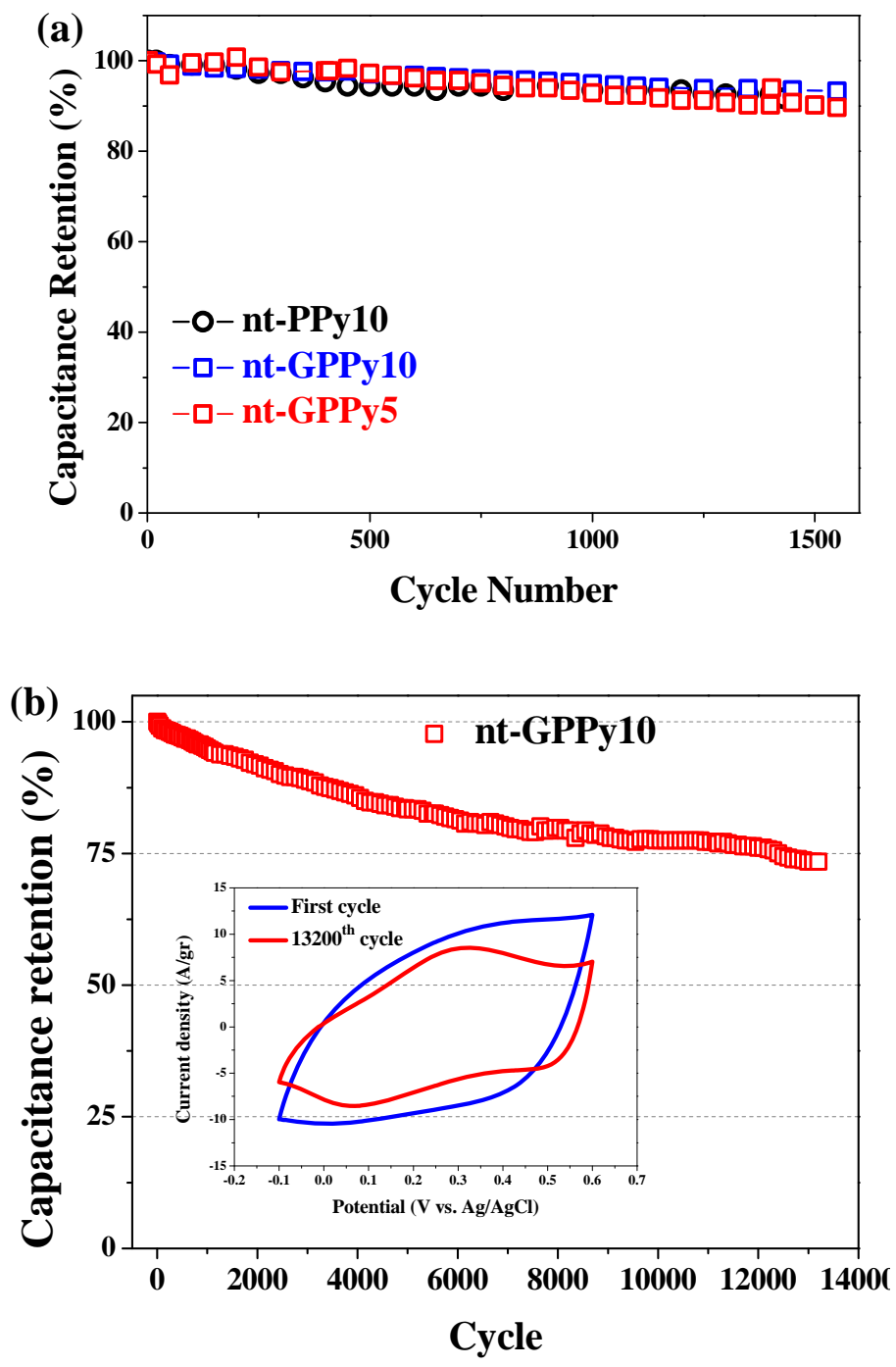

Figure 5 Cycling performance of nanotubular electrodes in $1 \mathrm{M} \mathrm{HClO}_{4}$ electrolyte at scan rate of $50 \mathrm{mV} \mathrm{s}^{-1}$. (a) Capacitance retention of nt-PPy10, nt-GPPy5 and nt-GPPy10. Specific capacitances of nt-GPPy5, nt-GPPy10 and nt-PPy10 decrease to $91.6,93$ and $94.5 \%$ of their original values after 1500 cycles respectively. (b) Capacitance retention of nt-GPPy10 after 13200 cycles. Specific capacitance is reduced to $75 \%$ after 13200 cycles at $50 \mathrm{mV} \mathrm{s}^{-1}$. Inset shows CV curve of first and $13200^{\text {th }}$ cycle. 

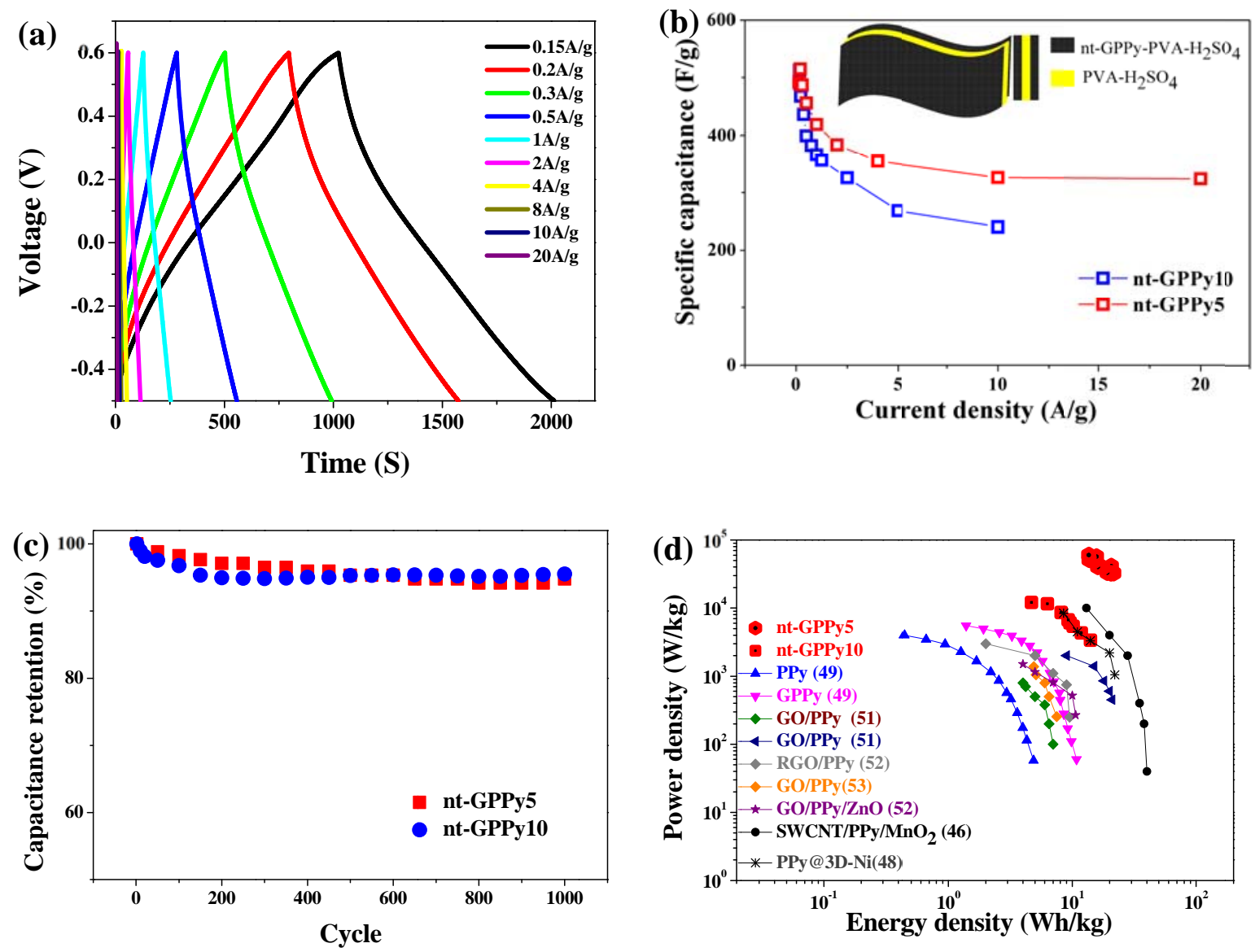

Figure 6 Solid state supercapacitance performance of nt-GPPy5 and nt-GPPy10 integrated with PVA- $\mathrm{H}_{2} \mathrm{SO}_{4}$ solid electrolyte. (a) Charge-discharge curves of nt-GPPy5 as a solid state supercapacitor at different current densities. (b) Specific capacitance versus current density of the symmetric solid state supercapacitor assembled by nt-GPPy5 and nt-GPPy10. Inset shows a schematic of the solid state supercapacitor. (c) Capacitance retention of nt-GPPy5 and nt-GPPy10. (d) Power density versus energy density of nt-GPPy5 and nt-GPPy10 and recently reported data from literature. 


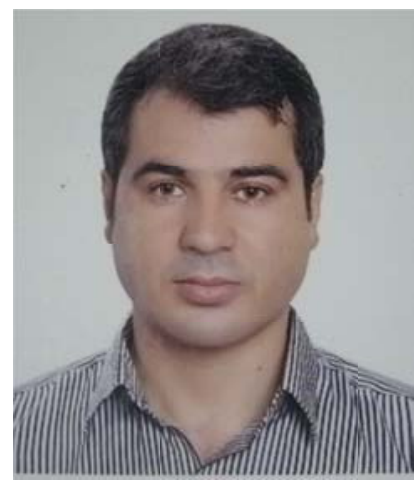

HamzehKashani is $\mathrm{PhD}$ student at Tohoku University, Japan. He received his Master degree in Materials Science in 2004 from University of Tehran, Iran. His research interest focuses on the synthesis, mechanical and electrochemical properties of nanoporous materials.

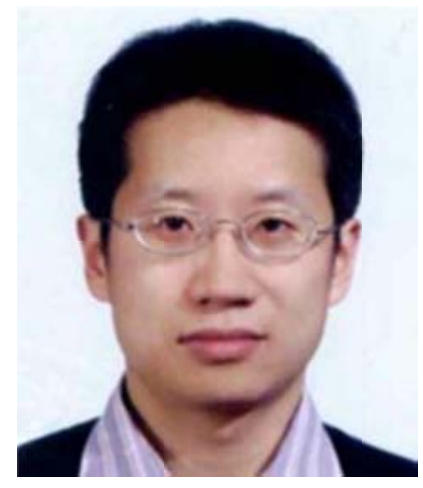

Luyang Chen is an assistant professor at Advanced Institute for Materials Research (AIMR), Tohoku University. He received a $\mathrm{PhD}$ degree in Inorganic Chemistry from University of Science and Technology of China, China, 2006. After that he worked as a postdoctoral fellow inAIMR, Tohoku University, Japan. His research interests include nanoporous metals and their applications, synthesis of carbon nanotubes and ultra high temperature ceramic nanomaterials.

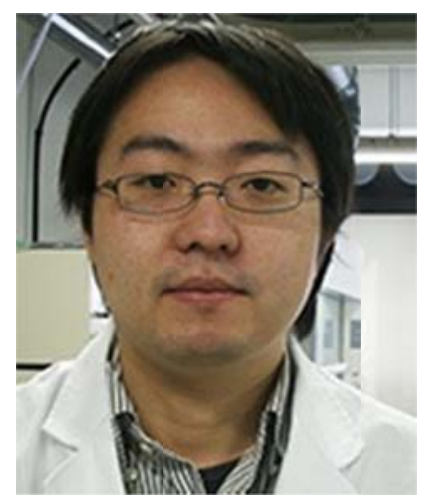

Yoshikazu Ito is an assistant professor at WPI-AIMR, Tohoku University, Japan. He received $\mathrm{PhD}$ degree from Tokyo Institute of Technology. His research interest include production of nanoparticles and graphene for energy related application. 


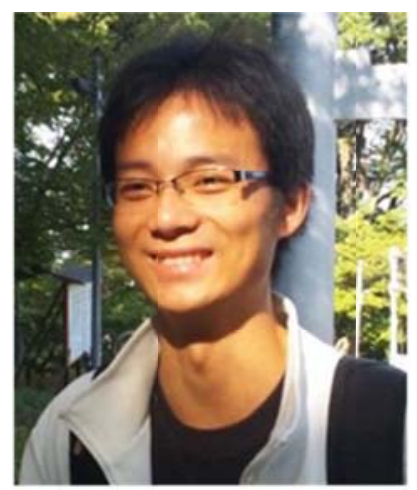

Jiuhui Han received his Master degree in Materials Science from Tohoku University in 2014. Now he is a Ph.D. candidate in Tohoku University and his current research focuses on the development and characterization of nanoporous materials for energy-related applications.

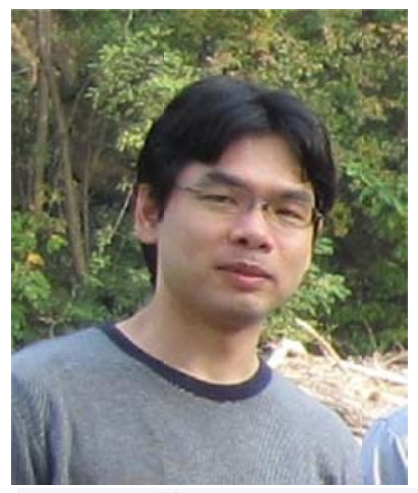

Akihiko Hirata received his $\mathrm{PhD}$ degree in Science and Engineering from Waseda University in 2003. His research interest include structural studies of alloy and intermetallic using Transmission Electron Microscopy and local atomic structure and crystallization process of metallic glasses.

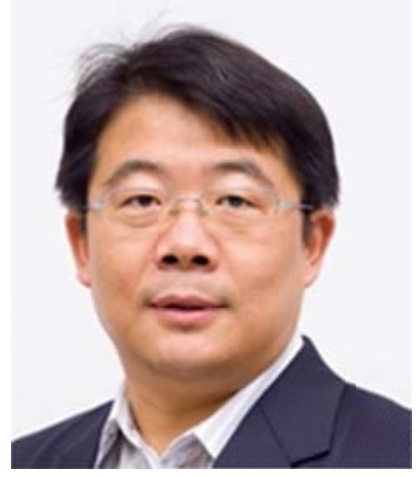

Mingwei Chen is a professor in AIMR and Materials Science department in Tohoku University, Japan and Shanghai Jiao Tong University, China. His research interests include structure-property relationship of advanced materials and the development and characterization of 3D nanoporous materials for energy and environment related applications. 


\section{Graphical Abstract}

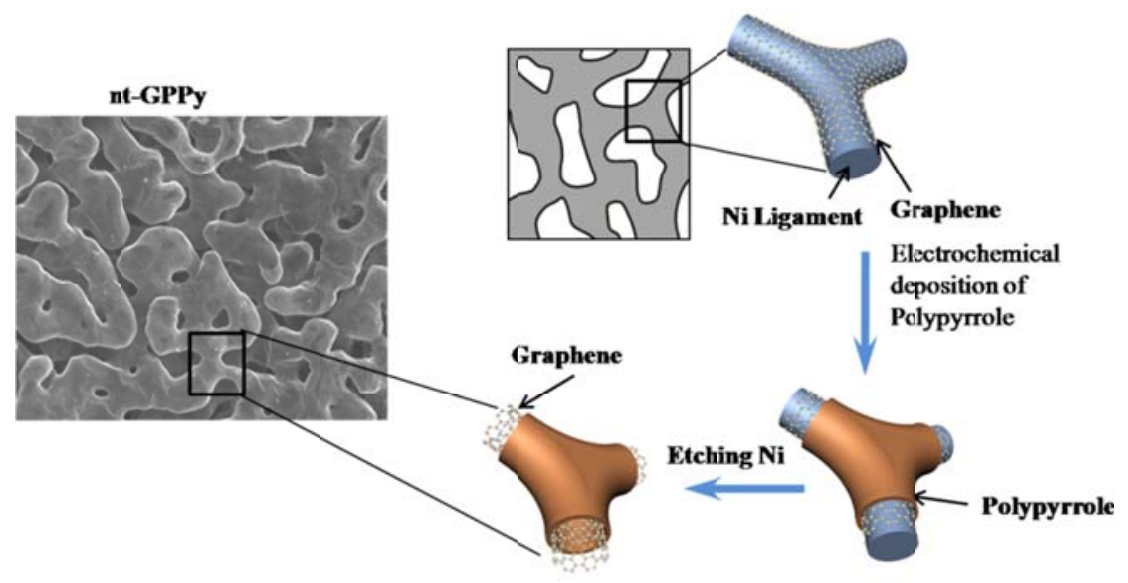

